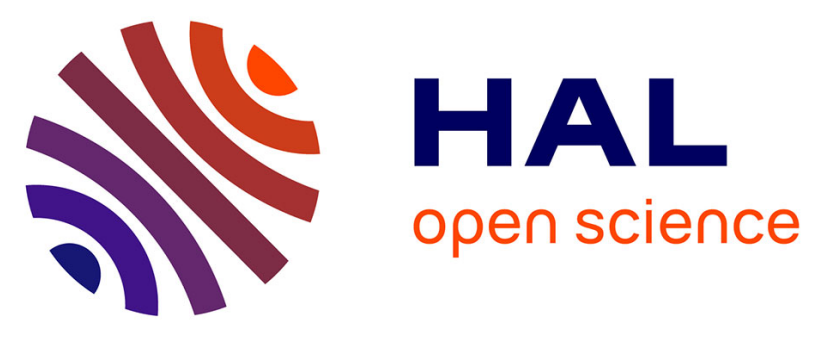

\title{
Temperature dependent photoluminescence and micromapping of multiple stacks InAs quantum dots
}

Ming Xu, Alexandre Jaffré, J Alvarez, Jean-Paul Kleider, Apichat Jittrong, Thitipong Chokamnuai, Somsak Panyakeow, Mohamed Boutchich, Songphol Kanjanachuchai

\section{To cite this version:}

Ming Xu, Alexandre Jaffré, J Alvarez, Jean-Paul Kleider, Apichat Jittrong, et al.. Temperature dependent photoluminescence and micromapping of multiple stacks InAs quantum dots. AIP Conference Proceedings, 2015, 1649, pp.3. 10.1063/1.4913536 . hal-01239179

HAL Id: hal-01239179

https://hal-centralesupelec.archives-ouvertes.fr/hal-01239179

Submitted on 11 Mar 2020

HAL is a multi-disciplinary open access archive for the deposit and dissemination of scientific research documents, whether they are published or not. The documents may come from teaching and research institutions in France or abroad, or from public or private research centers.
L'archive ouverte pluridisciplinaire $\mathbf{H A L}$, est destinée au dépôt et à la diffusion de documents scientifiques de niveau recherche, publiés ou non, émanant des établissements d'enseignement et de recherche français ou étrangers, des laboratoires publics ou privés. 


\section{AIP $\mid$ Proceedings}

Temperature dependent photoluminescence and micromapping of multiple stacks InAs quantum dots

Ming Xu, Alexandre Jaffré, José Alvarez, Jean-Paul Kleider, Apichat Jittrong, Thitipong Chokamnuai, Somsak Panyakeow, Mohamed Boutchich, and Songphol Kanjanachuchai

Citation: AIP Conference Proceedings 1649, 3 (2015); doi: 10.1063/1.4913536

View online: http://dx.doi.org/10.1063/1.4913536

View Table of Contents: http://scitation.aip.org/content/aip/proceeding/aipcp/1649?ver=pdfcov

Published by the AIP Publishing

Articles you may be interested in

Surface photovoltage and photoluminescence excitation spectroscopy of stacked self-assembled InAs quantum dots with InGaAs overgrown layers

J. Appl. Phys. 103, 084303 (2008); 10.1063/1.2907406

Photoluminescence of surface InAs quantum dot stacking on multilayer buried quantum dots Appl. Phys. Lett. 89, 243124 (2006); 10.1063/1.2408653

Temperature-dependent photoluminescence from patterned InAs quantum dots formed using metalorganic chemical vapor epitaxy

J. Appl. Phys. 99, 033503 (2006); 10.1063/1.2165415

Enhancement of room-temperature photoluminescence in InAs quantum dots

Appl. Phys. Lett. 83, 4300 (2003); 10.1063/1.1623324

Anomalous temperature dependence of photoluminescence from InAs quantum dots

J. Appl. Phys. 88, 2529 (2000); 10.1063/1.1288231 


\title{
Temperature dependent photoluminescence and micromapping of multiple stacks InAs quantum dots
}

\author{
${\underline{\text { Ming }} \mathrm{Xu}^{1}}^{1}$,, Alexandre Jaffré ${ }^{1}$, José Alvarez ${ }^{1}$, Jean-Paul Kleider ${ }^{1}$, Apichat \\ Jittrong $^{2}$, Thitipong Chokamnuai ${ }^{2}$, Somsak Panyakeow ${ }^{2}$, Mohamed Boutchich ${ }^{1}$ and \\ Songphol Kanjanachuchai ${ }^{2}$
}

\author{
${ }^{1}$ LGEP, CNRS UMR8507, SUPELEC, Univ Paris-Sud, Sorbonne Universités - UPMC, Univ Paris 06, 11 rue Joliot- \\ Curie, Plateau de Moulon, 91192 Gif-sur-Yvette Cedex, France \\ ${ }^{2}$ Semiconductor Device Research Laboratory (Nanotec Center of Excellence), Department of Electrical \\ Engineering, Faculty of Engineering, Chulalongkorn University, Bangkok 10330, Thailand

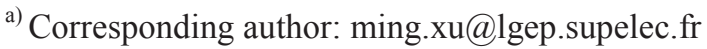

\begin{abstract}
We utilized temperature dependent photoluminescence (PL) techniques to investigate 1, 3 and 5 stack InGaAs quantum dots (QDs) grown on cross-hatch patterns. PL mapping can well reproduce the QDs distribution as AFM and position dependency of QD growth. It is possible to observe crystallographic dependent PL. The temperature dependent spectra exhibit the QDs energy distribution which reflects the size and shape. The inter-dot carrier coupling effect is observed and translated as a red shift of $120 \mathrm{mV}$ on the [1-10] direction peak is observed at $30 \mathrm{~K}$ on 1 stack with regards to 3 stacks samples, which is assigned to lateral coupling.
\end{abstract}

\section{INTRODUCTION}

Semiconductors III-V QDs are crystalline nano objects that are attractive for a wide range of detection related applications particularly in QD laser ${ }^{1}$, near infrared region (NIR) ${ }^{2}$ and high efficiency solar cells ${ }^{3}$. These nanostructures are typically grown by Molecular Beam Epitaxy (MBE) using the Stranski-Krastanow (SK) growth mechanism ${ }^{4}$ and their small size give them interesting tunability. Indeed, the low dimensionality implies that the peak emission of QDs can be adjusted by tuning the size, composition size and passivation to suit dedicated applications. On the other hand, these objects permit the study of the transport properties of single electrons as well as advanced heterojunctions for new concepts. One major challenge remains the integration of III-V QDs on silicon substrate to develop low cost silicon photonics devices. To investigate such complex nanostructures photoluminescence has proven to be an effective tool to monitor the electronic structure as well as the optical transitions.

In this study, we employed temperature dependent macro PL characterization to investigate InAs QDs on InGaAs cross hatched patterns (CHPs) grown by MBE on (001)-GaAs substrates ${ }^{5}$. The structure investigated is composed of multi stack QD of 1, 3 or 5 stacks of InAs QDs and 10-nm GaAs spacer. To better understand this QDs structure, it is also important to investigate not just macroscopic PL, but also the position-dependent PL or PL mapping. For that purpose we utilized a confocal micro PL system to map the sample at room temperature and observe the crystallographic dependence of the peak emission. The temperature dependant PL spectra exhibit major differences between samples due to different QD size and lateral coupling. The PL mappings reveal position dependent luminescence ${ }^{6}$. These characterizations contribute to improve the understanding of the structure's optical properties, and provide a basis for future improvements on QDs based optoelectronic devices. 


\section{SAMPLE PREPARATION}

\section{Quantum Dots Growth}

The QDs are grown on (001) GaAs substrates with MBE. After 300nm of GaAs buffer layer, the $\mathrm{In}_{\mathrm{x}} \mathrm{Ga}_{1-\mathrm{x}} \mathrm{As} \mathrm{CHP}$ layer of 25-nm is firstly deposited on the substrate, followed by $10 \mathrm{~nm}$ GaAs spacer layer then the QDs. Spacer and QDs deposition is repeated to form 3 and 5 stacks. At last, a 100nm capping layer of GaAs is deposited as passivation. The deposition of $10 \mathrm{~nm}$ GaAs spacing layer and QDs is repeated to obtain 3 stacks and 5 stacks. Further details on the growth are reported in ${ }^{5}$. Another set of sample have been fabricated without capping to carry out AFM measurements. The final structure is shown in Fig.1 (a).

\section{Morphology}

AFM imaging demonstrates the QDs distribution on the topmost layer. As is shown in Fig.1 (b), the QDs are aligned along [1-10] and [110] dislocation lines while some of them distribute randomly for 1 stack. The diameter of QDs for the 1 stack sample is $30 \mathrm{~nm}$. For 3 and 5 stacks, almost all of the QDs align themselves along dislocation lines. Their morphology depends on the growth direction, the shape of the 1 stack QDs is circular but those of the 3 stacks and 5 stacks are prolonged along [1-10] direction. In contrast, the size of 3 stacks and 5 stacks are 35nm along [110] and 60nm along [1-10]. In addition, as the [1-10] QDs began to grow prior to the [110], and to free-standing ones, their sizes are a little different, that is, $[1-10]>[110]>$ free-standing ${ }^{7}$, whereas the size on both directions are similar on multiple stack samples. The distance between QDs also varies with stack number. This inter-dot distance is a key parameter to favor coupling between QDs ensembles 5 . The lateral coupling rapidly decreases as the distance increases.

(a)

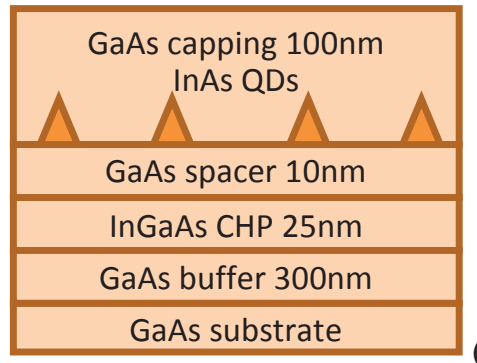

(b)

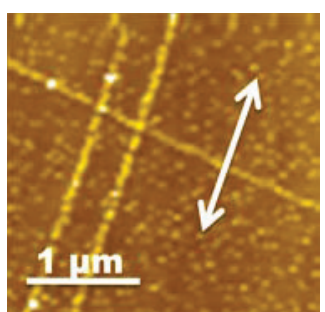

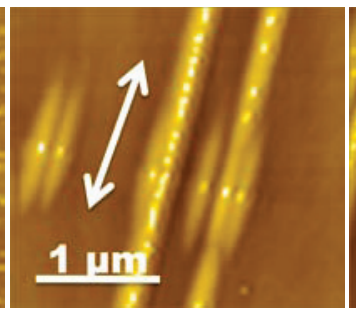

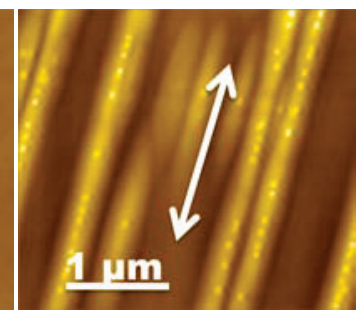

FIGURE 1. Sample structure for 1 stack sample and AFM imaging for 1, 3 and 5 stacks. Arrow in the images indicates the [1-10] direction.

\section{TEMPERATURE DEPENDENT PHOTOLUMINESCENCE}

\section{Measurement System}

Our PL system is equipped with a cryostat driven by helium circulation which allows cooling down until $20 \mathrm{~K}$. The excitation laser beam is projected onto the sample though cryostat window using optical fiber. The emitted luminescence is collected and focused into the monochromator and amplifier that convert photon flux to electrical signal. By controlling the monochromator, the PL spectrum is measured with a resolution of 25nm. The PL is measured under various temperatures between $30 \mathrm{~K}$ and $300 \mathrm{~K}$, with a step of $10 \mathrm{~K}$. The sample is clamped onto the cryostat and illuminated with a $785 \mathrm{~nm}$ laser, under a power density of $120 \mathrm{~mW} / \mathrm{cm}^{2}$. This power density permits only the ground state emissions. We performed several rounds of temperature measurements, from high to low as well as from low to high, to make sure that the measurement is stable and no hysteresis appears. 
(a)

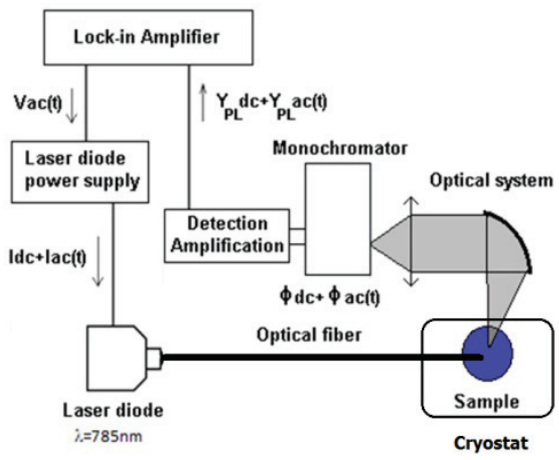

FIGURE 2. Schematic of our temperature dependent PL system

The micro PL measurements were performed with a frequency-doubled $532 \mathrm{~nm}$ NdYag laser operating in continuous mode as the excitation source up to $20 \mathrm{~mW}$ output power. The spectra were recorded through a $300 \mathrm{~mm}$ imaging spectrometer equipped with both 600 lines $/ \mathrm{mm}$ and 1800 lines $/ \mathrm{mm}$ gratings, and a back-illuminated CCD. A $100 \times$ dry and oil immersion objective with respectively a numerical aperture (NA) of 0.9 and 1.4 , and a $50 \mu \mathrm{m}$ core diameter multimode fiber acting as a pinhole were used to collect the signal. The expected lateral spatial resolution in the $x-y$ plane can reach $150 \mathrm{~nm}(\mathrm{NA}=1.4)$ and $250 \mathrm{~nm}(\mathrm{NA}=0.9)$. A particular attention was paid to the incident laser power density to avoid local heating effects. A hyperspectral mapping of a $20 \mu \mathrm{m}$ by $20 \mu \mathrm{m}$ area of the sample is made possible by exciting the sample surface with $532 \mathrm{~nm}$ laser and measuring the PL spectrum while scanning the surface using a piezoelectric stage with a spatial resolution of $10 \mathrm{~nm}$.

\section{Results}

The macro PL spectra are shown in fig.2. We observe that at room temperature PL intensity is as weak as noise level, which is negligible compared to those at low temperatures. At lower temperature emission peaks begin to emerge.

(a)

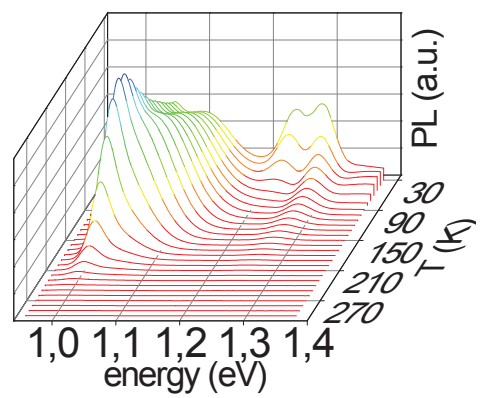

(b)

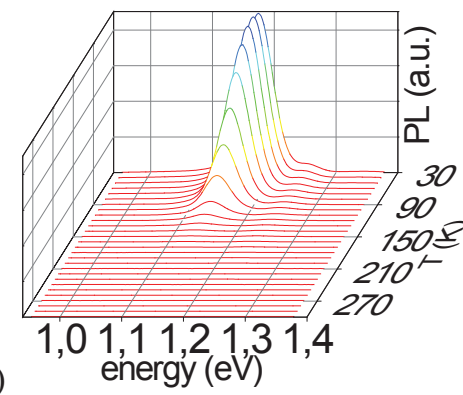

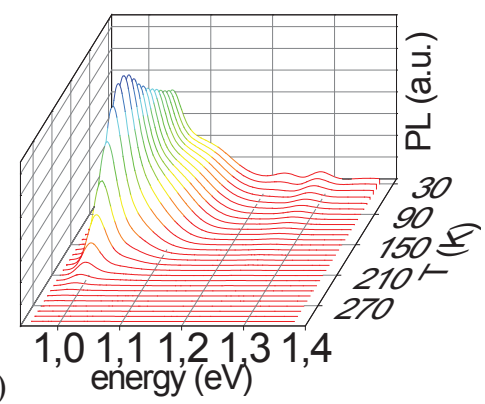

FIGURE 3. Temperature dependence of PL for (a) 1 stack (b) 3 stacks and (c) 5 stacks. The PL is measured every $10 \mathrm{~K}$ from $30 \mathrm{~K}$ to $300 \mathrm{~K}$.

Strong emissions minimize weaker ones when plotted in the same scale as is the case in Fig.3. We select some PL spectrums under various temperatures and rescaled them as shown in Fig.4 to give a clearer view of the emission peaks and the variation with temperature. 

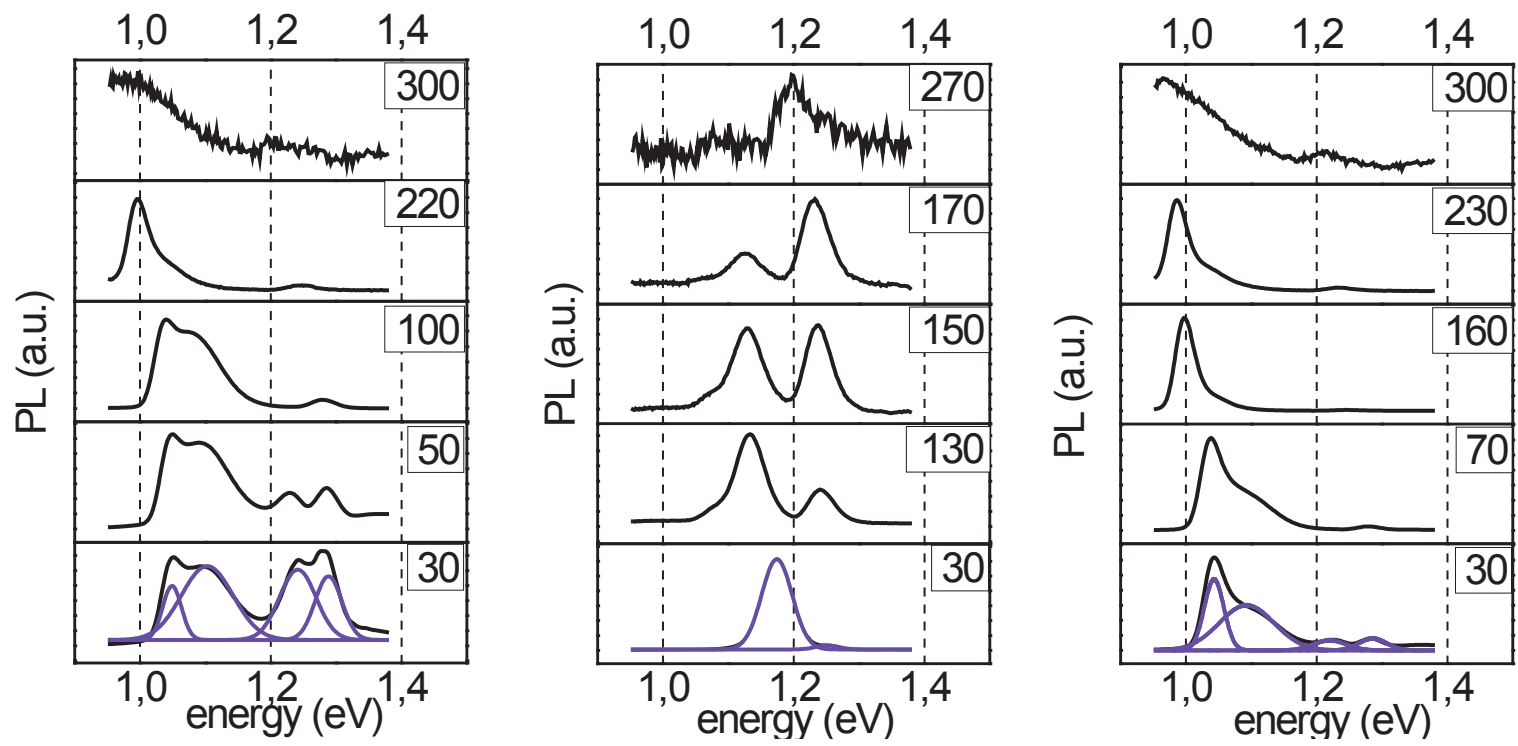

FIGURE 4. PL spectrum at some representative temperatures for 1 stack, 3 stacks and 5 stacks. Multiple Gaussian curve fitting is done for $30 \mathrm{~K}$ to indentify peak energy. 1 stack four peaks: $1.05,1.10,1.24,1.29 \mathrm{eV} ; 3$ stacks two peaks: $1.17,1.25 \mathrm{eV} ; 5$ stacks four peaks: $1.04,1.09,1.22,1.28 \mathrm{eV}$

In addition to temperature dependent measurement under standard PL system, we carried out micro mapping at room temperature in order to investigate the PL distribution along the various directions. Although the PL signal is relatively low at room temperature the mapping system has enough sensitivity to resolve the PL emission from noise and the images acquired confirm the QDs distribution across specific crystallographic orientations on the surface of 1 stack sample as shown in Fig.5.

(a)

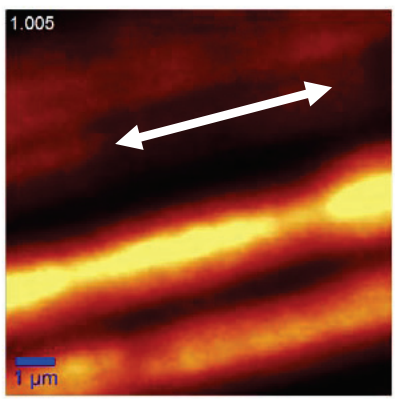

(b)

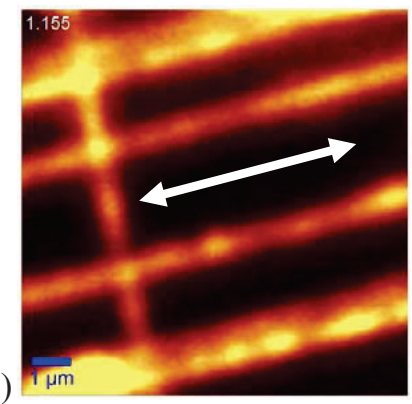

(c)

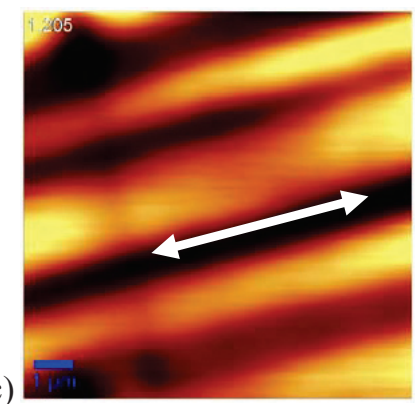

FIGURE 5. PL mapping of 1 stack at room temperature at (a) $1.005 \mathrm{eV}$ emission from [1-10] dots (b) $1.155 \mathrm{eV}$ from both [1-10] and [110] dots (c) $1.205 \mathrm{eV}$ from free-standing dots emission. Arrow indicates [1-10] direction.

\section{DISCUSSION}

In all samples, the emission peak of GaAs $\sim 1.42 \mathrm{eV}$ is not detected, indicating that the carriers generated in the GaAs and the wetting layer necessary for the growth are transferred to the QDs layer. We didn't observed vertical coupling as the spacing layer is thick enough to eliminate this effect between layers in multi-stack sample. However, strong lateral coupling is found for 1 stack.

For 1 stack, we find 4 emission peaks at $30 \mathrm{~K}$ as is shown in Fig.4 (a). In Fig.5 the first peak is located at 1.05eV, the second at $1.10 \mathrm{eV}$, the third at $1.24 \mathrm{eV}$ and the fourth at $1.29 \mathrm{eV}$. They correspond to emission of [1-10], [110] free-standing QDs and $\operatorname{In}_{\mathrm{x}} \mathrm{Ga}_{1-\mathrm{x}}$ As CHP layer respectively ${ }^{7}$. Moreover, compared to 3 stacks, 1 stack has smaller QDs size but lower ground state energy in Fig4.(a)-(b). This is characterized by a red shift of $120 \mathrm{mV}$ on the [1-10] direction peak is observed at $30 \mathrm{~K}$ when comparing 1 stack to 3 stacks samples. This shift is assigned to lateral coupling as discussed in ${ }^{8}$ when the dots are very close to each other as indicated in AFM imaging. 
Due to lack of free-standing QDs, the 3 stacks and 5 stacks show little of the corresponding emission. The 3stack spectrum has two peaks and when temperature increases they both decrease as a result of thermal quenching. The intensity of the first broad peak decreases much more rapidly than the second one, making the second peak only visible at relatively higher temperature as is shown in Fig.5 (b). At $30 \mathrm{~K}$, the first peak is at $1.17 \mathrm{eV}$ and the second at $1.25 \mathrm{eV}$. In fact the first peak is comprised of two closely located peaks that correspond to [1-10] and [110] dots respectively which are very close in size and shape as discussed above about Fig1.(b). The distances between QDs are bigger than 1stack, far enough not to induce coupling effect.

The behavior of 5 stacks is not similar to that of 3 stack in terms of spectra although their AFM morphology in Fig.1(b) quite resemble to each other. Inhomogeneity caused during the sample growth may be responsible for this discrepancy.

Due to the detection sensitivity, we get a noisy measurement result of 1 stack at room temperature as is shown in Fig.4(a). There is a wide peak at about $1.0 \mathrm{eV}$ and a small one at $1.2 \mathrm{eV}$. However, micro-mapping in Fig.5 demonstrates that $1.0 \mathrm{eV}$ emission comes from [1-10] QDs and that although we cannot see in macro-PL spectrum, [110] QDs are contributing to $1.155 \mathrm{eV}$ emission as shown in Fig.5(b). The $1.2 \mathrm{eV}$ emission should be attributed to free-standing QDs by comparing Fig.5(c) to (a) and (b).

\section{CONCLUSION}

We observed the temperature dependence of photoluminescence of multiple stack InGaAs quantum dots fabricated with MBE. The emission peaks are characteristic of the various QDs patterns present in the multi stack. In addition, using micro PL mapping, it is possible to resolve the emission peak along the crystallographic orientations and therefore identify the different QDs patterns. A blue shift of $120 \mathrm{meV}$ is observed on the [1-10] direction peak at $30 \mathrm{~K}$ between the 3 and 5 stacks sample, this is assigned to the lateral coupling between dots.

\section{ACKNOWLEDGMENTS}

Authors wish to thank the French Ministère des affaires étrangères et européennes (MAEE) and the Centre National de la Recherche Scientifique (CNRS) for their financial support in the STIC ASIA program, and Thailand Research Fund (RSA5580015), as well as China Scholarship Council (CSC).

\section{REFERENCES}

${ }^{1}$ A. Lee, Q. Jiang, M. Tang, A. Seeds, and H. Liu, Opt. Express 20, 22181 (2012).

${ }^{2}$ J.O. Kim, S. Sengupta, A.V. Barve, Y.D. Sharma, S. Adhikary, S.J. Lee, S.K. Noh, M.S. Allen, J.W. Allen, S. Chakrabarti, and S. Krishna, Appl. Phys. Lett. 102, 011131 (2013).

${ }^{3}$ Y. Shoji, K. Akimoto, and Y. Okada, J. Phys. Appl. Phys. 46, 024002 (2013).

${ }^{4}$ D. Leonard, M. Krishnamurthy, C.M. Reaves, S.P. Denbaars, and P.M. Petroff, Appl. Phys. Lett. 63, 3203 (1993).

${ }^{5}$ T. Chokamnuai, P. Rattanadon, S. Thainoi, S. Panyakeow, and S. Kanjanachuchai, J. Cryst. Growth 378, 524 (2013).

${ }^{6}$ W. Favre, J.-P. Kleider, D. Muñoz, S. Martin-de-Nicolás, and P.-J. Ribeyron, Phys. Status Solidi C 8, 775 (2011).

${ }^{7}$ T. Limwongse, S. Panyakeow, and S. Kanjanachuchai, Phys. Status Solidi C 6, 806 (2009).

${ }^{8}$ P. Yu, W. Langbein, K. Leosson, J.M. Hvam, N.N. Ledentsov, D. Bimberg, V.M. Ustinov, A.Y. Egorov, A.E. Zhukov, A.F. Tsatsul'nikov, and Y.G. Musikhin, Phys. Rev. B 60, 16680 (1999). 